\title{
Llano Alonso, Fernando H. Homo excelsior. Los límites ético-jurídicos del transhumanismo, Valencia, Tirant lo Blanch, 2018
}

\author{
Ignacio Campoy Cervera \\ Departamento de Filosofía del Derecho \\ Universidad Carlos III de Madrid
}

Fecha de recepción 01/10/2019 I De publicación: 15/06/2020

El libro Homo excelsior. Los límites ético-jurídicos del transhumanismo, del Catedrático de Filosofía del Derecho de la Universidad de Sevilla Fernando Llano, representa un excelente ejemplo de la adaptación de los conocimientos propios de la Filosofía del Derecho a las cuestiones morales relevantes que plantean las siempre cambiantes sociedades humanas.

En este sentido, el libro del profesor Llano se encuentra en una línea de comprensión de lo que es la Filosofía del Derecho que me parece plenamente acertada. Su libro responde a la idea de que la Filosofía del Derecho, lejos de ser una rama del conocimiento anquilosada, que ha de responder sempiternamente a los mismos temas con una misma metodología, tiene un carácter totalizador, trascendental, racional y crítico que le permite, le obliga, a ampliar su objeto de conocimiento a toda reflexión que tenga por objeto el material jurídico y, en concreto, desde la rama de la Teoría de la Justicia, a atender a las cuestiones básicas sobre cómo hacer una regulación jurídica que responda a los valores y principios de la Justicia, que en nuestras sociedades democráticas están centradas en garantizar la vida humana digna, de manera que a través del reconocimiento y protección efectiva del ejercicio de los derechos humanos, el mayor número de personas posible consiga alcanzar al máximo nivel posible el libre desarrollo de su propia personalidad.

Los temas que se abordan en el libro de referencia son algunos de los principales retos a los que se ha de enfrentar la humanidad en un futuro tan próximo que en realidad ya es presente. Sé que la expresión "algunos de los principales retos a los que se ha de enfrentar la humanidad" es usado tantas veces, e incluso con excesiva ligereza, que es difícil que consiga llamar la atención que merece del lector. No obstante, lo utilizo aquí porque realmente estoy convencido de que en este caso la expresión es completamente pertinente, e incluso, quizás con la excepción del cambio climático y todo lo que el mismo ha de conllevar, creo que las cuestiones que se tratan pueden ser los principales retos a los que 
se ha de enfrentar la humanidad entendida en su conjunto; porque de lo que se trata es, intentando resumirlo en pocas palabras, nada menos que de determinar cómo hemos de afrontar la inevitable interconexión entre el avance científico-técnico con la vida humana, en un momento en que, por primera vez en la historia, existe la posibilidad cierta de que la aplicación de esos conocimientos científico-técnicos produzca una transformación, sin vuelta atrás, de la propia naturaleza del ser humano.

Sin embargo, a pesar de la enorme trascendencia de las cuestiones que se plantean, y a las que el propio desarrollo de los conocimientos científico-técnicos asegura que se va a terminar dando una respuesta, aunque sólo sea de facto, es todavía claramente insuficiente el debate existente entre la comunidad científica (entendida en un sentido amplio) y la propia sociedad sobre cuáles han de ser esas respuestas que hemos de dar. Y dentro de esa escasez de respuestas, llama la atención la casi nula atención que se le ha prestado a estos temas en la Filosofía del Derecho española ${ }^{1}$; cuando la Filosofía del Derecho ha de ofrecer la necesaria perspectiva, desde el análisis crítico, que permita encauzar las respuestas que se den dentro del modelo de los derechos humanos, que, como antes indicaba, actualmente constituyen el núcleo de justicia de nuestras sociedades.

Es cierto que son de una enorme complejidad las cuestiones que para la humanidad plantea el actual avance de los conocimientos científicos y técnicos; así como que precisamente la escasa aportación que al debate se ha hecho hasta ahora, desde la sociedad en general y desde la Filosofía del Derecho en particular, hace que sea difícil atreverse a afrontar esas cuestiones desde el ámbito filosófico jurídico. Por eso, dada la trascendente y urgente necesidad de dar respuestas desde el modelo de los derechos humanos, las aportaciones que se realizan en esta línea deben de ser fomentadas y celebradas; y ése es el caso del libro del profesor Llano.

En realidad, el Catedrático de la Universidad Hispalense no parecía, en principio, debido a los temas a los que se había dedicado a lo largo de su brillante carrera académica, la persona de la que

\footnotetext{
${ }^{1}$ Más allá del ámbito de la bioética, en donde sí que se puede encontrar una mayor aportación, son muy escasas las aportaciones que se han realizado en relación directa con los temas del transhumanismo, la inteligencia artificial y la robótica. Aunque entre esas escasas aportaciones cabe destacar algunas muy valiosas, como son los libros: De Asís Roig, Rafael, Una mirada a la robótica desde los derechos humanos, Dykinson, Madrid, 2015; Pérez Luño, A.-E., Los derechos humanos en la sociedad tecnológica, Universitas, Madrid, 2012; y De Lucas Martín, Francisco Javier, Blade Runner. El Derecho, guardián de la diferencia, tirant lo blanch, Valencia, 2003; así como la obra colectiva Ballesteros, J. y Fernández, E. (coords.), Biotecnología y posthumanismo, Thomson Reuters Aranzadi, Navarra, 2007; el artículo de De Asís Roig, Rafael, "Robótica, Inteligencia Artificial y Derecho", Revista de Privacidad y Derecho Digital, vol. 3, núm. 10, 2018; y los dos de Pérez Triviño, José Luis, "X-Men: de mutantes y posthumanos. Ingeniería genética y pánico moral”, InterseXiones, núm. 3, 2012 y "Equality of Access to Enhancement Technology in a Posthumanist Society”, Dilemata, núm. 19, 2015.
} 
cabía esperar que se aventurase a tratar esta nueva y compleja temática. El profesor Llano había venido dedicando sus principales esfuerzos en construirse una sólida formación humanista a través del estudio del pensamiento de cuatro grandes pensadores: Guido Fassò, Inmanuel Kant, José Ortega y Gasset y Marco Tulio Cicerón ${ }^{2}$. Sin embargo, es posible que su decisión para decidirse a escribir este libro se debiera precisamente a esa formación humanista, al sentir la necesidad que hay de dar respuesta a esos grandes retos que plantean los avances científicos y técnicos, que él podía percibir en mayor medida como una amenaza cierta para la concepción y la defensa de la dignidad humana que se ha venido realizando desde la Modernidad y de la que él siempre ha sido un firme defensor. Sea ésa la motivación $\mathrm{u}$ otra, en todo caso, nosotros tenemos razones de sobra para celebrar que se decidiese a realizar este libro, porque la formación humanista del profesor Llano, lejos de ser una dificultad para abordar estos temas, ha supuesto una gran ventaja; siendo uno de sus grandes atractivos y logros haber conseguido aportar la necesaria visión a los problemas y soluciones que se ofrecen al transhumanismo, la inteligencia artificial, la robótica y la medicina genética, desde la defensa de los valores del humanismo.

Conforme a lo que ya he apuntado, se ha de entender que existen multitud de problemas que para el ser humano y las sociedades en que vive se pueden derivar del progreso en el conocimiento de las ciencias y las técnicas. En algún sentido se podría decir que esto siempre ha sido así, que es algo consustancial a la evolución de las sociedades humanas. No obstante, en el momento presente hay suficientes elementos que nos permiten afirmar que la humanidad (en su conjunto) está frente a una encrucijada absolutamente decisiva, que poco o nada tiene que ver con las decisiones tomadas en los momentos anteriores. Y la enorme diferencia es que, por primera vez en la historia, la humanidad se enfrenta a la posibilidad cierta de que, fruto de sus avances científico-técnicos, pueda o inevitablemente vaya a cambiar al ser humano en características que consideramos tan constitutivas de su esencia como es la mortalidad, la corporeidad o la individualidad.

Ante esta nueva y apremiante realidad, el libro del profesor Llano tiene la gran cualidad de ofrecer, simultáneamente, por una parte, una buena panorámica general de las principales cuestiones a las que se han de dar respuesta, y, en este sentido, las expone desde una perspectiva analítica y con

\footnotetext{
${ }^{2}$ A cada uno de estos autores dedicó años de investigación, entre cuyos principales frutos habría que destacar los libros: El pensamiento iusfilosófico de Guido Fassò, Tecnos, Madrid, 1997; El humanismo cosmopolita de Inmanuel Kant, Dykinson, Madrid, 2002; El Estado en Ortega y Gasset, Dykinson, Madrid, 2011; y El gobierno de la razón: la filosofía jurídico-política de Mario Tulio Cicerón, Aranzadi, Madrid, 2017.
} 
espíritu casi de divulgación científica, centrándose en los retos del transhumanismo, la inteligencia artificial, la robótica y la medicina genética; y por otra, un análisis de las respuestas que ahora se vislumbran a esos grandes retos, respecto a los que Fernando Llano deja siempre claro cuál es su propia posición personal, desde una óptica coherente con su formación humanista -a la que antes me refería y que resulta sumamente valiosa y enriquecedora-, que se muestra no sólo en los valores y conceptos teóricos desde los que se acerca a todos los problemas y soluciones planteadas, sino también en la propia metodología de análisis, donde adquieren gran relevancia las concepciones de los autores clásicos, como, de forma principal, su admirado Ortega y Gasset, pero también las continuas referencias a la literatura, la mitología y la filmografía occidental con que ilustra sus análisis.

De hecho, si lo que en última instancia está debatiéndose (aunque no sólo, claro) es la posibilidad de cambiar la propia esencia del ser humano conforme al propio avance de la ciencia humana, nada mejor que dejar claro cuál es la otra gran construcción de la cultura humana que se enfrenta directamente a ese posible resultado: la cultura humanista, la valoración de la dignidad del ser humano, la defensa de los derechos esenciales que hemos considerado que todo ser humano tiene por su propia condición de ser humano: los derechos humanos. Y es esa confrontación la que nos presenta el libro Homo excelsior. Los límites ético-jurídicos del transhumanismo, pues los límites que han de establecerse a ese posible desarrollo transhumanista, que se levanta animado por nuestros propios conocimientos ante la humanidad como un nuevo y magnífico o monstruoso Leviathan, han de encontrarse, nuevamente, en la propia esencia de la humanidad, en su cultura humanista, en su dignidad inviolable, en los derechos humanos.

El Catedrático de la Universidad de Sevilla identifica el transhumanismo como un "movimiento cultural e ideológico que propone el mejoramiento tecnológico de las capacidades físicas y cognitivas del ser humano hasta el punto de intentar alcanzar la perfección de su especie” (p. 17). No obstante, esa línea de actuación tiene límites inciertos, de manera que podría entenderse que el transhumanismo abarca todo conocimiento científico-técnico que va encaminado a alcanzar ese objetivo de la perfección del ser humano, pero también su superación, en tanto que no sería sólo perfeccionar al ser humano tal cual lo conocemos, sino que para algunos autores también llevará a superar esta fase de la evolución de la humanidad y configurar una "humanidad superior". Un ideal, o para algunos una casi inevitable realidad, que pretende ser alcanzado por ciertos desarrollos de la robótica, la inteligencia artificial y la medicina genética, que son analizados en el libro. 
En esa línea, hay que entender que los robots, como señala el catedrático de Filosofía del Derecho de la Universidad Carlos III de Madrid Rafael De Asís, son “máquinas cada vez más inteligentes que pueden ser capaces de captar sensaciones y que algunos afirman que en el futuro podrán estar dotados de conciencia propia. Además de los tradicionales robots industriales [...] en la actualidad, órganos humanos son sustituidos por robots, creándose prótesis artificiales que desempeñan funciones humanas o que incrementan facultades. También han aparecido los robots que prestan servicios, algunos de los cuales son robots personales [...] La combinación de la robótica con otros campos científicos $\mathrm{y}$, de forma especial con las neurociencias y la nanotecnología, permite posibilidades hasta hace poco sólo imaginables"3. Esto supone que a través de la robótica se podrá llegar, entre otras muchas posibilidades reales, a la configuración de ciborgs u hombres fusionados inescindiblemente con máquinas. De esta manera, la robótica se une con la inteligencia artificial, que en un momento álgido supondrá alcanzar la "singularidad", es decir el momento en que la inteligencia creada por los hombres será superior a la humana -una inteligencia que para algunos tendrá consciencia y autonomía propia-, o alcanzar la fusión de la inteligencia de nuestros cerebros con las redes de inteligencia artificial creadas. Un panorama que habría que completar con las posibilidades que también abrirá la medicina genética, tema que el profesor Llano trata en el correspondiente capítulo, aunque finalmente sólo se centra en "los pacientes cuya herencia genética les hace potencialmente transmisores de enfermedades graves o incurables" (p. 193).

El transhumanismo es, pues, un movimiento poliédrico, que podrá permitir la solución de problemas reales y acuciantes de los hombres ${ }^{4}$, pero que también puede mostrar su lado más amenazador $^{5}$. El profesor Llano afronta en su libro esas diferentes dimensiones del transhumanismo, advirtiendo de que "Cabe diferenciar tres variedades de transhumanismo: en primer lugar el transhumanismo cultural, inspirado en la crítica postmoderna [...] contra el humanismo y el proyecto de la modernidad; en segundo lugar, el transhumanismo biológico, que reivindica el ideal ilustrado de la perfectibilidad potencialmente infinita del ser humano [...] a través del biomejoramiento humano que persiguen las tecnociencias (fundamentalmente la biología y la medicina genética); por último, en

\footnotetext{
${ }^{3}$ En De Asís Roig, Rafael, Una mirada a la robótica desde los derechos humanos, cit., pp. 34-35.

${ }^{4}$ En este sentido, señala el profesor De Asís que "el transhumanismo es una filosofía que pretende emplear la tecnología para mejorar la vida de las personas, aumentar la inteligencia, y hacer a los seres humanos más felices y virtuosos. Parte del hecho de la posible mejora del ser humano a través de la tecnología" (op. cit., p. 51).

${ }^{5}$ Como también señala Rafael de Asís, "Se trata de un movimiento que posee distintas corrientes y que ha sido considerado por algunos como peligroso por atentar contra la esencia de lo humano" (op. cit., p. 52).
} 
tercer lugar, se encuentra el transhumanismo cibernético, que sería en realidad un auténtico posthumanismo, en la medida en que pretende crear una nueva especie a partir de la hibridación hombre/máquina (y que recurre preferentemente a la robótica y la IA más que a la biología)” ( p. 28).

En todo caso, si bien es muy importante el análisis de esas formas en que el movimiento transhumanista puede alcanzar sus objetivos básicos, resulta necesario entender antes qué implica ese objetivo último de la "perfección” del ser humano y si el mismo resulta o no deseable. Y para ello, conforme a lo que antes apuntaba, primero habría que entender que los postulados del transhumanismo no llevan sólo, o al menos no tienen que llevar sólo, a un desarrollo de las capacidades del ser humano hasta su máximo nivel posible -objetivo que, en realidad, es que el que se establece con el reconocimiento y efectiva protección de los derechos humanos-; sino que los postulados transhumanistas también conllevan, o al menos una parte de dichos postulados conllevan, dar un paso más allá del ser humano tal cual lo hemos conocido hasta ahora (Fernando Llano deja fuera de estas pretensiones al transhumanismo biológico, aunque no me parece que todos los planteamientos que caben en este tipo sean ajenos a dicha pretensión). Estos últimos postulados pretenderían, en última instancia, llegar a formar un "superhombre”, un ser más perfecto que el ser humano, en la medida en que el mismo no adolecería de todas las carencias, limitaciones o fallas que tenemos los seres humanos. Sin embargo, aunque dicho objetivo no puede dejar de mostrarse atractivo, el mismo ha de enfrentarse, con carácter previo a su posible consecución, con dos problemas esenciales, consecutivos lógicamente, y que de alguna manera cabría entender como irresolubles. El primero sería saber cuáles son esas carencias, limitaciones o fallas del ser humano, y quiénes y cómo las han de determinar; y el segundo sería saber por qué características se han de sustituir las anteriores, y quiénes y cómo las han de determinar.

Una primera aproximación a las cuestiones básicas antes planteadas podría apuntar a que si bien hay zonas de penumbra, sin embargo también hay alguna zona de claridad neta; por ejemplo, superar la condición mortal del ser humano parece que es una utopía que el ser humano ha buscado siempre (y si no se veía posible en la vida terrena al menos se ponía la confianza en la vida ultraterrena), así como evitar o superar las enfermedades es algo que también han procurado siempre todas las personas. Entonces, ¿no se podría considerar como objetivamente bueno que la persona no tenga necesariamente que morir y que no tenga ninguna enfermedad durante su vida, ser amortales y sanos, al menos mientras así lo deseemos? E incluso yendo un poco más allá, ¿no hay virtudes que en las sociedades 
humanas siempre hemos considerados positivas y, por lo tanto, deseables, como la valentía, la belleza, la inteligencia o la fuerza física?; ¿no sería, pues, también objetivamente bueno que las personas fuesen valientes, bellos, inteligentes y fuertes? Dicho de otra manera, ¿se puede pensar que haya alguien a quien ofreciéndosele la amortalidad, la salud, el valor, la belleza, la inteligencia y la fuerza no las aceptase?; ¿no sería injusto, pues, negarles esas posibilidades a las personas cuando se estuviese en condiciones de ofrecérselas?

Pero la realidad es que incluso en esas zonas de claridad hay zonas de penumbra, que las respuestas a las anteriores preguntas no son tan obvias como puede parecer. Y no lo son al menos por dos principales motivos. Primero, porque ninguna de esas características del ser humano (quizás aquí con la excepción de la mortalidad) son objetivas y claras, sino que, al menos en buena medida, no dejan de ser construcciones culturales de las sociedades humanas (¿no es claro que los estándares de belleza varían en las diferentes sociedades?, ¿no es evidente que algunas condiciones humanas se han considerado como enfermedades en diferentes sociedades y como signos de distinción positiva en otras?); pero también porque no está nada claro que constituya un ideal garantizar que los seres humanos logren cambiar su condición en el sentido indicado (¿es de verdad deseable que el ser humano no sea mortal, que no padezca ninguna enfermedad durante su vida, que responda a unos estándares establecidos de belleza o de fuerza o que tenga un nivel determinado de inteligencia?). Y en otra línea, pero de la máxima importancia para la justicia social, antes de responder que sí a las cuestiones antes formuladas, habría que plantearse, por una parte, si no es absolutamente necesario saber qué implicaciones tendrían esos cambios para la sociedad en aspectos tan esenciales como el reparto de los recursos alimenticios o energéticos; y por otra, si "objetivizar" unos estándares de belleza, fuerza o inteligencia, no supone que desde ahora nos estemos ya manejando con unos patrones culturales que implican que hay personas más "defectuosas" que otras, personas que, aunque sólo sean en el sentido de no adaptarse a los patrones y estándares establecidos de la excelencia humana, son "inferiores" a otras, con lo que eso implica de atentado contra su dignidad y derechos humanos. Reitero que éstas son las penumbras que se alzan en las zonas de claridad. Está claro que los seres humanos seguiremos deseando y luchando por alargar una vida sana, en la que se desarrollen, entre otras, nuestras capacidades físicas, estéticas e intelectuales; pero los límites y los ideales están lejos de ser claros y objetivos. Es más, no puede dejar de producirnos un inefable, pero profundo, malestar, pensar que finalmente se consiguiese alcanzar el objetivo de sustituir al ser humano por un "superhombre", pues es una utopía que no parece que pueda dejar de alcanzarse si no es como una terrible distopía. De hecho, 
creo que la utopía que nos debería mover ha de seguir siendo la utopía de los derechos humanos, que lo que nos exige es dirigir los nuevos conocimientos científico-técnicos a perfeccionar las sociedades humanas, de manera que consiguiésemos superar las sociedades discriminatorias que tenemos y construir unas sociedades inclusivas, en las que realmente fuese respetada la igual dignidad de todos y cada uno de los seres humanos.

En todo caso, es muy importante entender que todas estas cuestiones no sólo apuntan a un futuro, más o menos lejano, en el que haya que decidir qué tipo de humanidad queremos construir o proteger, no es sólo atender a un previsible punto de no retorno, que se alcanzaría en las próximas décadas, para la propia supervivencia o transformación del ser humano; sino que las mismas afectan a muy diferentes ámbitos hoy en día, en los que se plantean cuestiones diferentes pero directamente relacionadas con las anteriores, como son: ¿qué tipo de educación se ha de proporcionar a los niños ante las nuevas sociedades tecnológicas y qué fines ha de tener la educación teniendo en cuenta las posibilidades educativas que ofrecen las nuevas tecnologías?; ¿pueden los padres tomar decisiones sobre la selección genética que afecta a sus hijos?; ¿tenemos derecho a que no se utilicen nuestros datos personales por las grandes corporaciones que controlan Internet, incluso aunque los demos voluntariamente a través del correo electrónico o las redes sociales?; ¿es deseable la automatización de la función judicial?; etc. En este sentido, advierte lúcidamente el profesor Llano que "El Mac Guffin [con lo que alude al recurso narrativo que inventase Alfred Hitchcock y que el propio autor explica en las páginas anteriores] del posthumanismo consiste precisamente en presentar una situación hipotética, por lo general perteneciente al género de lo catastrófico, como una realidad alarmante más que probable. Este horizonte apocalíptico de guerras de exterminio entre ciborgs, robots y humanos mantiene la mente ocupada del público aficionado a la ciencia ficción o tendente a la hipocondría, para distraer su atención de lo esencial: por ejemplo, una cuestión tan relevante como la situación crítica de los derechos humanos en aquellas regiones del planeta donde la robótica es aplicada sin remilgos con propósitos a veces espurios y nada humanitarios (es el caso del uso abusivo de los drones o aeronaves sin tripulación con fines militares)" (pp. 135-136).

Resulta claro, pues, que hay que atender al presente, a la posibilidad y a la realidad de la utilización hoy día de los avances científicos y técnicos y su posible impacto en los derechos humanos, pero también, y a pesar de la contundencia del párrafo antes citado del profesor Llano, no hay que pensar (tampoco lo hace, ni mucho menos, el Catedrático de la Universidad de Sevilla) que tener que 
darles la mayor importancia a esas cuestiones actuales ha de disminuir en lo más mínimo la necesidad urgente de pensar en las respuestas que hay que dar para evitar esos futuros posibles y pavorosos y construir, en cambio, un futuro deseable para la humanidad. De nuevo, se trata de evitar los dos peligros, tan malo sería para nuestras sociedades no evitar el Escila de la utilización de los conocimientos científico-técnicos en contra de los derechos humanos hoy día, como no tomar las medidas necesarias para evitar el Caribdis de una sociedad futura en la que queden anulados los derechos humanos y la consiguiente dignificación del ser humano.

En el libro del profesor Llano encontramos información muy valiosa para entender los ideales y las corrientes de pensamiento que entretejen este difícil entramado actual, sin obviar el análisis de relevantes políticas y normativa europea y nacional al respecto; las conexiones existentes entre el transhumanismo y la filosofía postmoderna, y su contraposición con la filosofía ilustrada y universalista; el determinismo científico y el determinismo tecnológico, y su contraposición con el indeterminismo y la valoración de la autonomía individual; el antropocentrismo y el tecnocentrismo cientificista; o la técnica y su interrelación con la naturaleza humana, desde ángulos tan diferentes como los que proporcionan el transhumanismo, los bioconservadores o el humanismo tecnológico, que el profesor Llano ve representado de la mejor manera en el pensamiento de Ortega y Gasset. Un análisis que, como antes apuntaba, realiza el profesor Llano desde una aproximación crítica en la que prevalece la revalorización del humanismo, la dignidad humana y los derechos humanos.

Finalizo esta recensión casi como la comencé, recomendando vivamente la lectura del libro Homo excelsior. Los límites ético-jurídicos del transhumanismo, porque el mismo es, sin duda, una valiosa contribución a la necesaria reflexión sobre las cuestiones aquí señaladas. Querámoslo o no, seamos conscientes o no, la humanidad se encuentra ante una encrucijada fundamental, en las que las respuestas a las cuestiones decisivas que plantean el transhumanismo, la singularidad de la inteligencia artificial, el "perfeccionamiento" humano a través de ciborgs o de la medicina genética, se van a dar, se están en realidad ya dando, sí o sí. Y esas respuestas pueden producirse tras un debate amplio, público, abierto e informado, en el que se decida cuál ha de ser la regulación que fomente, limite o encauce los conocimientos científico-técnicos, para procurar que siempre prevalezcan los valores esenciales de la humanidad; o puede ser por la vía de los hechos, en la que los mismos se impongan a través de un desarrollo imparable guiado por grandes corporaciones y los intereses económicos y políticos a los que respondan. En una famosa escena de la película The Matrix (de 1999), Morfeo le da la oportunidad a 
Neo de elegir entre una ignorancia placentera con la que continuaría siendo esclavo de las máquinas o un conocimiento que le llevaría al enfrentamiento con las mismas por una incierta libertad, advirtiéndole de que es su última oportunidad, que la decisión que tome no tiene vuelta atrás. Es muy posible que también nosotros nos encontremos ante decisiones que van a determinar de manera definitiva el presente y el futuro de la humanidad, en un nivel y con una trascendencia sin precedentes, ¿no será, pues, necesario, abrir al debate amplio, público, abierto e informado las cuestiones esenciales que se nos plantean y las diferentes respuestas que podemos darles? 